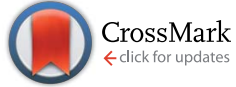

Cite this: RSC Adv., 2017, 7, 20

Received 18th October 2016 Accepted 6th December 2016

DOI: 10.1039/c6ra25422e

www.rsc.org/advances

\section{The 3D mechanical environment and chemical milieu influence the hMSC fibrogenesis and fibroblast-to-myofibroblast transition $\uparrow$}

\author{
Ruodan $\mathrm{Xu}{ }^{a}$ Flemming Besenbacher ${ }^{\mathrm{b}}$ and Menglin Chen ${ }^{* a b}$ \\ The chemical cytokine milieu and the mechanical environment are two central elements in the \\ development and progression of fibrosis. In this study, we demonstrated that, compared to a stiff \\ substrate, a fibrinogen based hydrogel scaffold provided a 3D microenvironment for enhanced human \\ mesenchymal stem cell (hMSC) proliferation, embedded connective tissue growth factor (CTGF) for \\ directed fibrogenesis, and a compliant substrate for alleviated myofibrogenesis.
}

\section{Introduction}

The major challenges for stem cell therapy are cell survival and directed cell differentiation. ${ }^{1}$ An extracellular matrix (ECM) provides both physical (stiffness, size, shape, and thickness) and biochemical (e.g. cell adhesive ligands) cues to regulate stem cell functions. ${ }^{2,3}$ Engineering three-dimensional (3D) stem cell microenvironments for optimal in vitro culture conditions is a determinant factor to realize the efficacy of the therapy.,

Connective-tissue cells contain multiple types of cells differentiated from mesenchymal stem cells (MSCs) and play a central part in the support and reparation of almost every tissue and organ. ${ }^{6}$ MSCs transplantation is, however, at risk of turning into myofibroblasts leading to pathogenic fibrosis rather than regenerative tissues, such as when injected into the myocardial scar after infarct, ${ }^{7}$ when delivered to interstitial fibrosis ${ }^{8}$ and when engrafting in a murine model of chronic lung fibrosis. ${ }^{9}$ Therefore MSC transplantation with prevention of fibrosis progression is critical for the success of regenerative therapy.

Differentiation of myofibroblasts in the presence of central profibrotic cytokine transforming growth factor- $\beta 1$ (TGF- $\beta 1$ ) is intended to rapidly restore the mechanical integrity of damaged tissues. Both macroscopically tissue stiffening and subcellular cytoskeletal contractions/tensions are found related to latent TGF- $\beta 1$ activation and subsequent progression of fibrosis. ${ }^{10}$ Measured with the subcellular precision of atomic force microscopy (AFM), normal heart muscle, vascular smooth muscle and skeletal muscle exhibit Young's modulus of approximately $10 \mathrm{kPa} .{ }^{11}$ In contrast, fibrotic tissue was found to

\footnotetext{
${ }^{a}$ Department of Engineering, Aarhus University, DK-8000 Aarhus C, Denmark ${ }^{b}$ Interdisciplinary Nanoscience Center (iNANO), Aarhus University, DK-8000 Aarhus C, Denmark.E-mail: menglin@inano.au.dk

$\dagger$ Electronic supplementary information (ESI) available. See DOI: 10.1039/c6ra25422e
}

be stiff, with Young's moduli of $100 \mathrm{kPa}$, which is close to the stiffness of collagen-dense tendon or the osteoid structure. ${ }^{12}$

Hydrogels are soft biomaterials with stiffness in the same range of normal tissues. Here, we investigate the fibrogenesis of hMSCs in a three-dimensional (3D), connective tissue growth factor (CTGF) loaded, PEGylated fibrinogen (PF) based scaffold (abbreviated as PFP-C) $)^{13}$ under external TGF $\beta$-1 stimulation at different time points, and compare it with 2D stiff TCP substrate with CTGF administrated in the culture medium (denoted as TCPc). The in vitro compatibility of hMSCs was evaluated by measuring cell viability/cytotoxicity. The differentiation profile was studied in 4 weeks' duration using immunocytochemistry and quantitative real-time PCR.

\section{Materials and methods}

\subsection{Fabrication of scaffolds}

The preparation of the scaffold followed the similar procedure at a previous study. ${ }^{13}$ Briefly, $100 \mu \mathrm{L}$ fibrinogen-PEG (PF) hydrogel (with photoinitiator) loaded with CTGF (40 ng) was added onto thin $(100 \mu \mathrm{m})$ electrospun PCL $(1 \mu \mathrm{m}$ in diameter $)$ mesh and kept at $4{ }^{\circ} \mathrm{C}$ overnight in the dark. The PCL mesh was used to improve the mechanical integrity of the scaffold. The scaffold (height, $1 \mathrm{~mm}$ ) was formed after exposing under longwave UV light ( $365 \mathrm{~nm}, 4-5 \mathrm{~W} \mathrm{~cm}^{-2}$ ) for 3-4 minutes to perform the cross-linking. The cross-linked scaffolds were stable during the 4 weeks' in vitro culture.

\subsection{Cell culture}

Human mesenchymal stem cells (hMSCs, Lonza Walkersville, Walkersville, MD, USA) were expanded in growth medium containing high glucose Dulbecco's Modified Eagle Medium (DMEM-HG, Gibco, UK) containing $10 \%$ fetal bovine serum (FBS, Biowhittaker, Walkersville, MD) and $1 \%$ penicillinstreptomycin (P/S, Gibco, Grand Island, NY) at $37{ }^{\circ} \mathrm{C}$ in 
Table 1 Primers used for real-time PCR

\begin{tabular}{lll}
\hline Gene & Primer sequence $\left(5^{\prime}-3^{\prime}\right)$ & $T_{\mathrm{m}}\left({ }^{\circ} \mathrm{C}\right)$ \\
\hline \multirow{2}{*}{ FN1 } & Forward CGGAGAGACAGGAGGAAATAGCC & 71 \\
& Reverse TTGCTGCTTGCGGGGCTGTC & 75 \\
\multirow{2}{*}{ FP1 } & Forward AGCTTCTTGGGGAAAAGGAC & 63.3 \\
& Reverse CCCCAACCACATCAGAGG & 64.2 \\
\multirow{2}{*}{ SSMA } & Forward CCGACCGAATGCAGAAGGA & 68.6 \\
& Reverse ACAGAGTATTTGCGCTCCGAA & 65.8
\end{tabular}

a humidified atmosphere of $5 \% \mathrm{CO}_{2}$. Cells were enzymatically treated with trypsin for passaging every 5-7 days when confluent. Passage 4 of hMSCs was used for cell seeding in scaffolds.

\subsection{Cell/scaffold construct culturing and differentiation}

$20 \mu \mathrm{L}$ of hMSCs $\left(5 \times 10^{5}\right.$ cells $)$ were dropped onto sterile PFP-C, and cells were allowed to attach for $1 \mathrm{~h}$ before the further addition of growth medium supplemented with $50 \mu \mathrm{g} \mathrm{mL}$ ascorbic acid (Sigma Aldrich, Schnelldorf, Germany). Cells were differentiated for a 2-4 week period, with conditioned medium change every third day. $10 \mathrm{ng} \mathrm{mL}{ }^{-1}$ TGF- $\beta 1$ was added for one week to induce differentiation towards myofibroblasts at week 2,3 and 4 .

\subsection{LDH assay}

As described in previous study, ${ }^{13}$ after 24 hours of hMSCs culturing on PFP-C, culture media was taken for LDH assay for determining damaged cells. The LDH Cytotoxicity Detection Kit was purchased from Roche Diagnostics (Mannheim, Germany) and the assay was performed according to the manufacturer's instructions. Absorbance was measured at $490 \mathrm{~nm}$ using a Victor X5 microplate reader (PerkinElmer Life and Analytical Sciences, Shelton, CT).

\subsection{Cell viability/proliferation study}

Cell viability/proliferation was determined by Cell Counting Kit8 (CCK-8, Dojindo, Kumamoto, Japan) analysis. After culturing cells in conditions of TCPc and PFP-C for 1, 7, and 14 days, cell medium was removed and filled with diluted CCK-8 reagent solution (CCK-8 reagent was diluted with fresh cell culture medium in a 1 to 10 ratio) in each well. The same volume of culture medium was used as the blank. After $2 \mathrm{~h}$ incubation of the CCK-8 reagent with cells, $100 \mu \mathrm{L}$ of mixture was collected and transferred into a new 96 well plate, the absorbance at $450 \mathrm{~nm}$ was measured for each well as above. WST-8 in CCK-8 is reduced by dehydrogenase in cytoplasm giving a yellow-color formazan dye. The intensity of the yellow color analyzed by a plate reader at $450 \mathrm{~nm}$ is directly corresponding to the number of living cells. Sextuplicate exposures of each sample were made under the same conditions. For calculation of proliferation index, absorbance values at a time point were normalized against day 1 absorbance values for the same group $\left(N_{\text {day } x} / N_{\text {day } 1}\right)$.

\subsection{RNA isolation and qPCR}

RNA was isolated from cells/PFP-C constructs using Trizol (Life technologies) following the manufacturer's instructions and the RNA yield was quantified using Nanodrop ND-1000 (NanoDrop Technologies, Inc.). Complementary DNA was synthesized
A

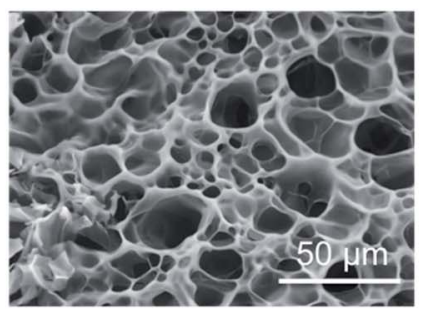

B

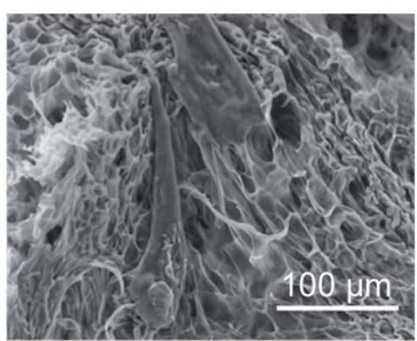

C

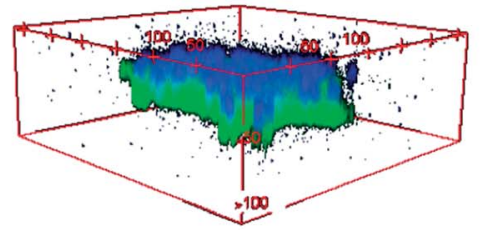

D

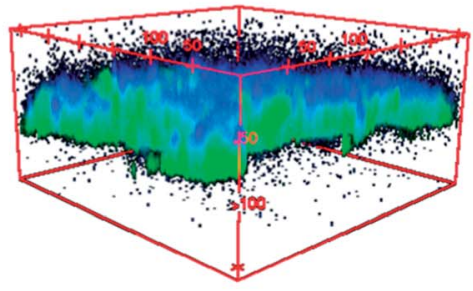

E

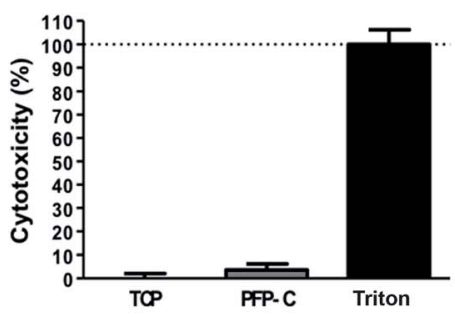

$\mathbf{F}$

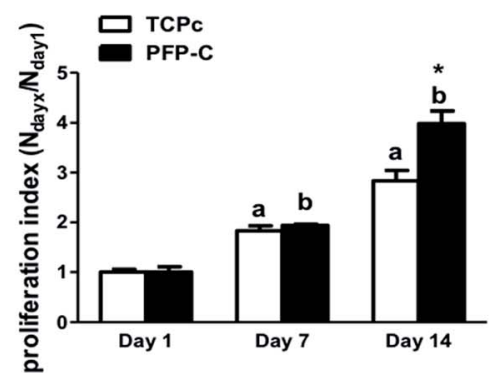

Fig. 1 SEM images and biocompatibility of PFP-C with hMSCs. (A and B) SEM images of the scaffold and cross-section view of hMSC-scaffold construct, respectively. (C and D) hMSCs infiltration in 3D scaffold by confocal z-stack imaging at day 1 and day 3, respectively. (E) LDH activity measured from the culture media collected 24 hour after hMSCs seeding on PFP-C scaffold. Low toxicity (\%) control was growth media. High toxicity control (\%) was from cells seeded on TCPC and incubated with $1 \%$ Triton X-100. (F) Quantitative analysis of 14 day proliferation index on TCPC and PFP-C nanocomposite, data were normalized on day 1 (a: stand for significant difference between samples and the control on TCPC on day 1 with $p<0.05$; b: stand for significant difference between samples and the control on PFP-C on day 1 with $p<0.05$; *: stand for significant difference between samples on PFP-C and TCPC on day 14). 
using High capacity RNA-to-cDNA kit (Applied Biosystems, Foster City, CA). The cDNA was stored at $-20{ }^{\circ} \mathrm{C}$ until further analysis. Real-time PCR was performed in a Lightcycler $480 \AA$ (Roche Diagnostics, Mannheim, Germany) using a SYBR Green kit (Roche) detection with primers listed in Table 1. Fold differences were calculated using the standard $2^{-\Delta \Delta C_{\mathrm{t}}}$ method with GAPDH as the housekeeping gene.

\subsection{Immunocytochemistry and fluorescence microscopy}

Cells/PFP-C constructs were fixed with $4 \%$ paraformaldehyde (PFA) in PBS for $15 \mathrm{~min}$ at room temperature followed by permeabilization $30 \mathrm{~min}$ with $0.5 \%$ Triton X-100. Cells were subjected to immunofluorescence staining with $\alpha$ SMA (1:400, Abcam, Cambridge, UK) antibody $4{ }^{\circ} \mathrm{C}$ overnight. The cells were then washed three times with PBS for 10 min each, and incubated with Alexa 594-labeled donkey anti-mouse secondary antibody (1:1000, Abcam) at room temperature for $1 \mathrm{~h}$. After washing with PBS, Hoechst 33258 ((1: 25 000) Life technologies, Carlsbad, CA) was added for $10 \mathrm{~min}$ incubation for nuclei staining. The cells/PFP-C constructs were visualized using a Zeiss LSM 700 laser confocal microscope (Carl Zeiss MicroImaging GmbH, Germany).

\subsection{Statistical analysis}

Data are presented as the means \pm standard error of mean (SEM). The statistical analyses were performed using Student's $t$-test for two-group comparison or a one-way repeated-measure analysis of variance test (ANOVA) to compare multiple data
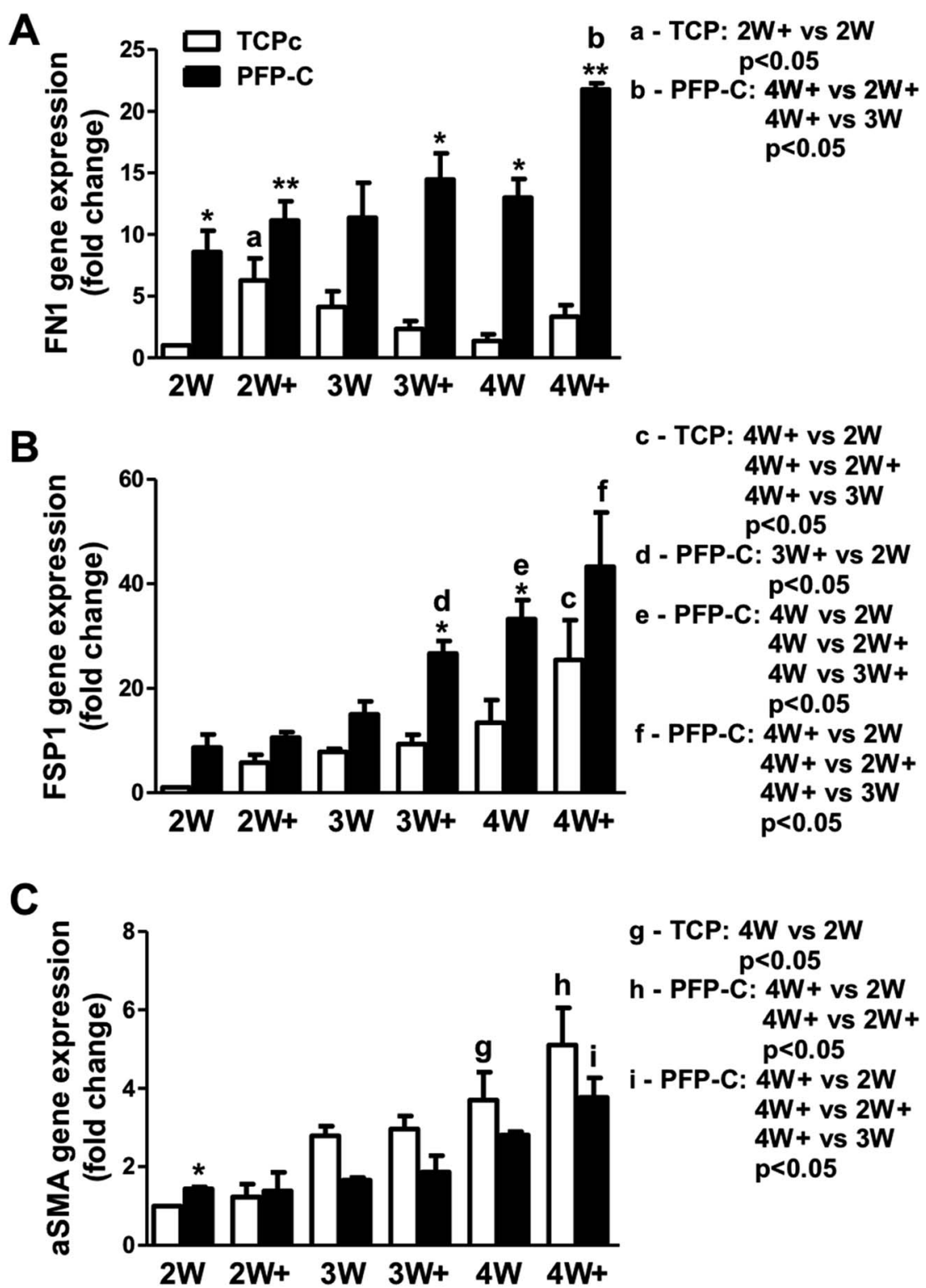

Fig. 2 Real-time PCR gene expression analysis of extracellular matrix and surface adhesion protein FN1 (A), fibroblast marker FSP1 (B) and myofibroblast marker aSMA (C) over 4 weeks (the breaking lines set at 1 are the gene expression levels of hMSC on TCPC at 2W) (* and **: stand for significant difference between samples on PFP-C and samples on TCPC at same time point with $p<0.05$ and 0.01 , respectively; a-i: ANOVA compare multiple data groups with $p<0.05$ ). 
groups, followed by Turkey's post hoc test. Values of $p<0.05$ were considered statistically significant. The software used for statistics and the creation of figures was Prism 5 (GraphPad, San Diego, CA, USA).

\section{Results and discussion}

\subsection{Cell proliferation on 3D PFP-C scaffold}

The 3D PFP-C scaffold with microporous structure (Fig. 1A) allowed hMSCs adhesion (Fig. 1B) and infiltration (Fig. 1C and D). The cytocompatibility of PFP-C to support hMSC cell adhesion and growth was evaluated by LDH and CCK assays. As shown in LDH assay (Fig. 1E), PFP-C only induced $5.4 \pm$ $2.1 \%$ cytotoxicity on hMSCs, compared to that of TCP control. In the CCK assay (Fig. 1F), PFP-C initially showed similar level of growth rates compared to TCPc at day 7, with no significant differences between two substrates. At day 14, the proliferation rate in PFP-C appeared significantly higher than that on TCPc, confirming PFP-C, exceeding TCPc as monolayer cell culturing substrate, provides a $3 \mathrm{D}$ cell culture environment.

\subsection{Fibroblast/myofibroblast differentiation of hMSCs on PFP-C scaffold}

The fibrogenic differentiation of hMSCs on PFP-C and TCPc and subsequent myofibrogenic differentiation were investigated at different time points ( 2 week, 3 week, 4 week), with $(2 \mathrm{~W}+, 3 \mathrm{~W}+$, $4 \mathrm{~W}+)$ or without $(2 \mathrm{~W}, 3 \mathrm{~W}, 4 \mathrm{~W})$ external TGF $\beta-1$ administration in the last week. An extracellular matrix and surface adhesion protein fibronectin (FN1), a fibrogenic marker fibroblast-

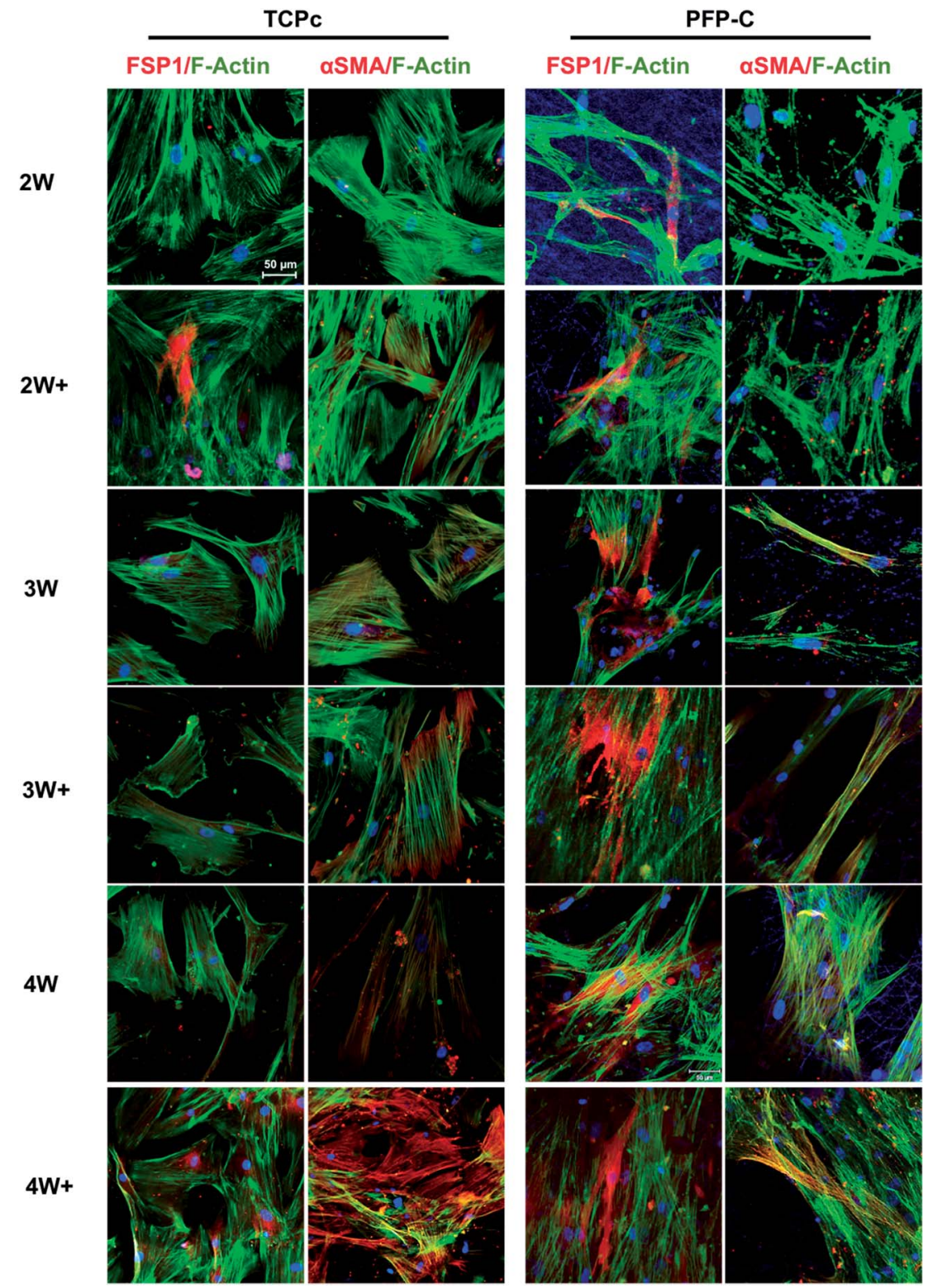

Fig. 3 Immunofluorescence analysis of fibroblast marker FSP1, myofibroblast marker aSMA over 4 weeks differentiation. Nuclei were stained with Hoechst (blue). Scale bar $=50 \mu \mathrm{m}$. 
specific protein 1 (FSP1), and $\alpha$ SMA as a myofibrogenic marker were employed as the characteristic genes. The results are presented in Fig. 2.

hMSCs cultured in PFP-C exhibited significantly elevated FN1 expression at most time points $(2 \mathrm{~W}, 2 \mathrm{~W}+, 3 \mathrm{~W}+, 4 \mathrm{~W}$ and $4 \mathrm{~W}+$ ) compared to that on TCPc, which confirmed that the hydrogel scaffold enhanced cell adhesion. The external TGF $\beta-1$ administration only significantly increased FN1 expression at week 2 on TCPc and at week 4 on PFP-C.

Cells on both substrates showed significant increased expression of FSP1 over the time, confirming the progression of fibrogensis. FSP1 expressions at $3 \mathrm{~W}+$ and $4 \mathrm{~W}$ on PFP-C had already shown similar amount of expressions compared with those on TCPc at $4 \mathrm{~W}+$, which were significantly higher than those in corresponding TCPc groups, which indicated that hMSCs started fibrogenic differentiation earlier and faster on PFP-C than those on TCPc.

No significant changes of $\alpha S M A$ expression were found in PFP-C groups suggested no progression of myofibrogenesis on PFP-C. In contrast, hMSCs on TCPc showed progression of myofibrogenesis, where cells at $4 \mathrm{~W}$ and $4 \mathrm{~W}+$ showed significantly higher expression of $\alpha S M A$ than those at $2 \mathrm{~W}, 2 \mathrm{~W}+$, respectively. The $\alpha S M A$ expression on PFP-C was significantly higher at $2 \mathrm{~W}$, but significantly lower from 3 weeks to 4 weeks than those on TCPc. The early expression of $\alpha S M A$ could be related to the early fibrogenesis on PFP-C; while there was no progression of myofibrogenesis suggesting PFP-C with stiffness $\left(3.62 \pm 0.38 \mathrm{kPa}\right.$, Fig. S1†) similar to normal muscle tissues, ${ }^{\mathbf{1 1}}$ alleviated myofibrogenesis. This is consistent with the previous founding that that TGF- $\beta 1$ only upregulates the myofibroblast marker $\alpha$-SMA in fibroblasts grown on stiff, but not on compliant, substrates. ${ }^{14}$

To characterize the protein expressions, the immunocytochemistry staining was used and fluorescence images were shown in Fig. 3. In general, hMSCs gradually differentiated towards fibroblasts on both TCPc and PFP-C, proven by increased protein expression of fibroblast marker FSP1. Consistent with gene expression, FSP1 expression appeared at week 2 on PFP-C and at week 3 on TCPc, suggesting the earlier

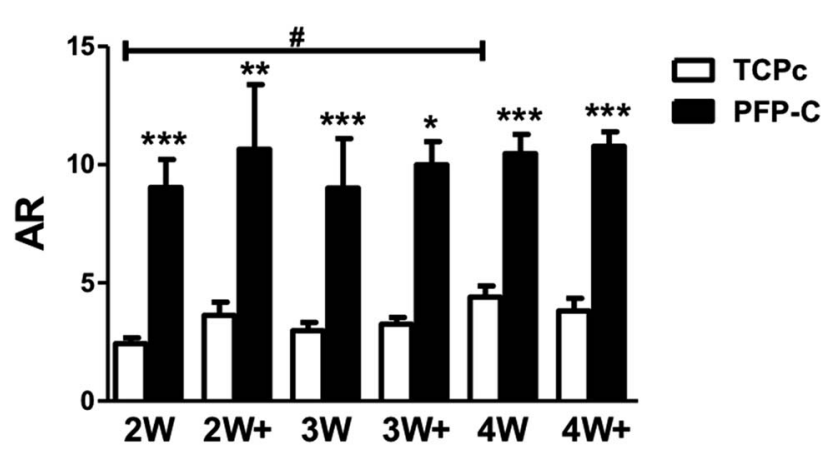

Fig. 4 Quantification of cell morphologies on TCPC and PFP-C over 4 weeks differentiation by AR. *,** and ***: stand for significant difference between samples on PFP-C and samples on TCPC at same time point with $p<0.05,0.01,0.005$, respectively; \#: stand for significant difference between samples on TCPC at $4 \mathrm{~W}$ and $2 \mathrm{~W}$ with $p<0.05$. fibrogenesis on PFP-C. It is noteworthy that cells on TCPc (Young's modulus around $1 \mathrm{GPa}$ ) showed much higher expression of $\alpha$ SMA than those on PFP-C in all conditions with or without administrating TGF $\beta-1$, confirming that matrix stiffness plays a central role in myofibrogenesis.

\subsection{Effect of PFP-C on cell morphology}

Many factors have been found to influence cell morphology and cell-cell contact, ${ }^{15}$ including growth factors, ${ }^{16}$ chemical characteristics of substrates, ${ }^{\mathbf{1 6}}$ and several physical cues such as stiffness, ${ }^{17}$ topography of substrate. ${ }^{18}$ Aspect ratio (AR) is an important measurement of cell shape. ${ }^{19}$ Higher aspect ratio validates a narrowed/shrunken but very elongated morphology. Using the images from immunocytochemistry staining, we analyzed aspect ratio (Fig. 4). Cells on PFP-C generally appeared significantly higher ARs compared to those on TCPc at all time points, which is consistent with the observations that cells on PFP-C became more polarized, migrated into the 3D matrix and formed highly spindled cell bodies that connected to each other. The cells on TCPc had no significant changes on ARs over the 4 weeks' culture, except those on $4 \mathrm{~W}$ were more spindleshaped and presented slightly higher AR value compared to those on 2W. Therefore, fibroblast morphology depended highly on substrate stiffness; more stress fiber bundles (lower $\mathrm{AR}$ ) were found on stiff substrate, which is consistent with previous studies. ${ }^{\mathbf{2 0 , 2 1}}$ As fibrinogen was reported with enhancing effect on hMSC proliferation ${ }^{22}$ but exerted no influence on hMSC differentiation, ${ }^{22,23}$ the $3 \mathrm{D}$ fibrinogen hydrogel mainly provided a compliant $3 \mathrm{D}$ matrix to alleviate myofibrogenesis.

\section{Conclusions}

In this study, we observed alleviated myofibrogenesis of hMSCs on 3D PFP-C hydrogel matrix, which provides 3D microenvironment for homing stem cells, embedded CTGF for directed fibrogenesis, and compliant substrate for maintaining TGF $\beta-1$ latency.

\section{Acknowledgements}

We gratefully acknowledge the funding for project ElectroMed (11-115313) from the Danish council for strategic research, Aarhus University Research foundation and Carlsberg Foundation for the financial support.

\section{References}

1 V. Tabar and L. Studer, Pluripotent stem cells in regenerative medicine: challenges and recent progress, Nat. Rev. Genet., 2014, 15, 82-92.

2 P. Lu, K. Takai, V. M. Weaver and Z. Werb, Extracellular Matrix Degradation and Remodeling in Development and Disease, Cold Spring Harbor Perspect. Biol., 2011, 3, a005058.

3 P. C. D. P. Dingal and D. E. Discher, Combining insoluble and soluble factors to steer stem cell fate, Nat. Mater., 2014, 13, 532-537. 
4 S. V. Murphy and A. Atala, 3D bioprinting of tissues and organs, Nat. Biotechnol., 2014, 32, 773-785.

5 W. L. Murphy, T. C. McDevitt and A. J. Engler, Materials as stem cell regulators, Nat. Mater., 2014, 13, 547-557.

6 B. J. A. Alberts and J. Lewis, et al., Molecular Biology of the Cell, Garland Science, New York, 4th edn, 2002.

7 M. Breitbach, T. Bostani, W. Roell, Y. Xia, O. Dewald, J. M. Nygren, et al., Potential risks of bone marrow cell transplantation into infarcted hearts, Blood, 2007, 110, 1362-1369.

8 V. Ninichuk, O. Gross, S. Segerer, R. Hoffmann, E. Radomska, A. Buchstaller, et al., Multipotent mesenchymal stem cells reduce interstitial fibrosis but do not delay progression of chronic kidney disease in collagen4A3-deficient mice, Kidney Int., 2006, 70, 121-129.

9 X. Yan, Y. Liu, Q. Han, M. Jia, L. Liao, M. Qi, et al., Injured microenvironment directly guides the differentiation of engrafted Flk-1(+) mesenchymal stem cell in lung, Exp. Hematol., 2007, 35, 1466-1475.

10 B. Hinz, Tissue stiffness, latent TGF-beta1 activation, and mechanical signal transduction: implications for the pathogenesis and treatment of fibrosis, Curr. Rheumatol. Rep., 2009, 11, 120-126.

11 R. G. Wells and D. E. Discher, Matrix elasticity, cytoskeletal tension, and TGF-beta: the insoluble and soluble meet, Sci. Signaling, 2008, 1, pe13.

12 A. J. Engler, C. Carag-Krieger, C. P. Johnson, M. Raab, H. Y. Tang, D. W. Speicher, et al., Embryonic cardiomyocytes beat best on a matrix with heart-like elasticity: scar-like rigidity inhibits beating, J. Cell Sci., 2008, 121, 3794-3802.

13 R. Xu, M. B. Taskin, M. Rubert, D. Seliktar, F. Besenbacher and M. Chen, hiPS-MSCs differentiation towards fibroblasts on a 3D ECM mimicking scaffold, Sci. Rep., 2015, 5, 8480.

14 P. D. Arora, N. Narani and C. A. McCulloch, The compliance of collagen gels regulates transforming growth factor-beta induction of alpha-smooth muscle actin in fibroblasts, Am. J. Pathol., 1999, 154, 871-882.

15 F. Guilak, D. M. Cohen, B. T. Estes, J. M. Gimble, W. Liedtke and C. S. Chen, Control of Stem Cell Fate by Physical Interactions with the Extracellular Matrix, Cell Stem Cell, 2009, 5, 17-26.

16 A. D. Whetton and T. M. Dexter, Influence of growth factors and substrates on differentiation of haemopoietic stem cells, Curr. Opin. Cell Biol., 1993, 5, 1044-1049.

17 A. J. Engler, S. Sen, H. L. Sweeney and D. E. Discher, Matrix Elasticity Directs Stem Cell Lineage Specification, Cell, 2006, 126, 677-689.

18 M. J. Dalby, N. Gadegaard and R. O. C. Oreffo, Harnessing nanotopography and integrin-matrix interactions to influence stem cell fate, Nat. Mater., 2014, 13, 558-569.

19 X. Yao, R. Peng and J. Ding, Effects of aspect ratios of stem cells on lineage commitments with and without induction media, Biomaterials, 2013, 34, 930-939.

20 X. Huang, N. Yang, V. F. Fiore, T. H. Barker, Y. Sun, S. W. Morris, et al., Matrix stiffness-induced myofibroblast differentiation is mediated by intrinsic mechanotransduction, Am. J. Respir. Cell Mol. Biol., 2012, 47, 340-348.

21 F. Liu, J. D. Mih, B. S. Shea, A. T. Kho, A. S. Sharif, A. M. Tager, et al., Feedback amplification of fibrosis through matrix stiffening and COX-2 suppression, J. Cell Biol., 2010, 190, 693-706.

22 J. D. Kisiday, B. W. Hale, J. L. Almodovar, C. M. Lee, M. J. Kipper, C. Wayne McIlwraith, et al., Expansion of mesenchymal stem cells on fibrinogen-rich protein surfaces derived from blood plasma, J. Tissue Eng. Regener. Med., 2011, 5, 600-611.

23 O. Schmidt, J. Mizrahi, J. Elisseeff and D. Seliktar, Immobilized fibrinogen in PEG hydrogels does not improve chondrocyte-mediated matrix deposition in response to mechanical stimulation, Biotechnol. Bioeng., 2006, 95, 1061-1069. 